\title{
3-Dimensional "Relativity" for Axisymmetric Stationary Space-Times
}

\author{
Z. PerJés \\ Central Research Institute for Physics, Budapest, Hungary
}

Received November 25, 1968

\begin{abstract}
The equivalence of the axisymmetric stationary vacuum gravitational field problem to a 3-dimensional "relativity theory" in the presence of a certain scalar matter field is shown. An invariant classification can be achieved with respect to the algebraic structure of the 3-dimensional trace-free Ricci-tensor. The extension of these results to electrovac spaces is also discussed.
\end{abstract}

\section{Introduction}

H. Levy [1] found recently a 3-dimensional stress tensor for axially symmetric stationary gravitational fields. The aim of the present work is a further development of this result to a 3-dimensional covariant formulation of the problem.

The basic concept will be discussed in Section 2. We shall show here that the axially symmetric stationary gravitational field problem is in vacuo completely equivalent to a 3 -dimensional relativity theory in the presence of a certain "matter field" and with axial symmetry.

A quite general property of the Einstein spaces subject to the condition

$$
R_{\mu \nu}-\frac{1}{2} g_{\mu v} R=T_{\mu v}
$$

and containing a Killing vector field is that they can equally well be described as $n-1$ dimensional Einstein spaces, at the expense of appearing some additional material fields. The particular class of symmetric Einstein spaces we picked out excels by the simple structure of the corresponding field equations.

In Section 3 we propose an invariant classification of the related space-times based on the algebraic properties of the 3-dimensional Ricci tensor. Section 4 deals with the electrovac problem. In the Summary we discuss the various new possibilities offered by our method for the study of the axially symmetric space-times. 


\section{Foundation of the 3-Dimensional "Relativity"}

The line element of an arbitrary axially symmetric vacuum gravitational field may be written [2]:

where

$$
\begin{aligned}
& d S^{2}=f^{-1} d s^{2}-f(d t+\omega d \Phi)^{2} \\
& d s^{2}=e^{2 \gamma}\left(d \varrho^{2}+d z^{2}\right)+\varrho^{2} d \Phi^{2}
\end{aligned}
$$

and $f, \omega, \gamma$ are functions of $x^{1}=\varrho$ and $x^{2}=z$ only. Using the notation of F. J. ERNst [3], we introduce the function $\varphi$ by

$$
\nabla \varphi=-f^{2} \varrho^{-1} \hat{n} \times \nabla \omega .
$$

Here $\nabla$ is the 3 -dimensional gradient operator and $\hat{n}$ stands for a unit vector pointing in the azimuthal direction. We remark that $\varphi$ agrees with Papapetrou's scalar function [4] $A$. For the field of a rotating source of finite extension, with mass $m$ and angular momentum $a \cdot m$ we have the following asymptotic conditions:

if $r=\left(\varrho^{2}+z^{2}\right)^{1 / 2} \rightarrow \infty$, then

$$
f \rightarrow 1-\frac{2 m}{r} ; \quad \phi \rightarrow \frac{a m z}{r^{3}} ; \quad \gamma \rightarrow-\frac{m^{2}}{2} \frac{\varrho^{2}}{r^{4}} .
$$

The field equations constitute two groups, the first of which is easily written down without referring to any particular coordinate system:

$$
\left.\begin{array}{l}
f \Delta f=\nabla f \nabla f-\nabla \varphi \nabla \varphi \\
f \Delta \varphi=2 \nabla f \nabla \varphi .
\end{array}\right\}
$$

$\triangle$ stands for the Laplace operator in 3-dimensional Euclidean space. The second group of the field equations determines $\gamma$ in terms of $f$ and $\varphi$ :

$$
\left.\begin{array}{l}
\gamma_{1} / \varrho=\frac{1}{4 f^{2}}\left(f_{1}^{2}-f_{2}^{2}+\varphi_{1}^{2}-\varphi_{2}^{2}\right), \\
\gamma_{2} / \varrho=\frac{1}{2 f^{2}}\left(f_{1} f_{2}+\varphi_{1} \varphi_{2}\right)
\end{array}\right\}
$$

(the suffixes denoting partial derivatives). The right hand sides of the system (6) can be written respectively as the $-T_{11}$ and $-T_{12}$ components of the 3 -dimensional symmetric tensor

$$
T_{i k}=-\frac{1}{2 f^{2}}\left\{f,_{i} f,_{k}+\varphi,_{i} \varphi,_{k}-\frac{1}{2} g_{i k}[(\nabla f \nabla f)+(\nabla \varphi \nabla \varphi)]\right\} .
$$

This tensor was found in a slightly different form first by $\mathrm{H}$. Levy [1]. He has shown that the divergence of $T_{i k}$ vanishes and stated that $T_{i k}$ has all the properties of a gravitational stress tensor. At this point it is natural to ask, whether a generalization of the definition (7) to a curved space $V_{3}$ exists. Then Eqs. (5) would appear as the "material field equations" in $V_{3}$ and Eqs. (6) would become the gravitational equations.

Choosing the line element (2) for $V_{3}$ and calculating

$$
G_{i k}=R_{i k}-\frac{1}{2} g_{i k} R \text {, }
$$


we get

$G_{11}=-G_{22}=-\gamma_{1} / \varrho ; G_{12}=-\gamma_{2} / \varrho ; G_{33}=-\varrho^{2} e^{-2 \gamma}\left(\gamma_{11}+\gamma_{22}\right)$,

the remaining components vanishing. Eqs. (6) turn out to be the (11) and (12) components of the gravitational equations

$$
G_{i k}=T_{i k},
$$

while $G_{33}=T_{33}$ is a consequence of the field Eqs. (5), (6). The definition (7) of $T_{i k}$ is preserved, but using now the line element (2). The covariant divergence of $T_{i k}$ again vanishes and the form of the field Eqs. (5) may be maintained changing the definition of the Laplace operator to

$$
\Delta f \stackrel{\text { def }}{=} g^{i j} f_{; i ; j} \text {. }
$$

Glancing at the line element (1), we see that our abstract space $V_{3}$ is equivalent to the hypersurfaces $d t+\omega d \Phi=0$ up to a conform factor $f^{-1}$.

Following the notation of ERNST, we introduce the complex "material field" in $V_{3}$ by writing

$$
\varepsilon=f+i \varphi .
$$

Then Eqs. (5), (6) may be written as follows

$$
\begin{aligned}
(\operatorname{Re} \varepsilon) \varepsilon^{; i} ; i & =\varepsilon^{; i} \varepsilon_{; i}, \\
R_{i k}-\frac{1}{2} g_{i k} R & =-\frac{1}{4(\operatorname{Re} \varepsilon)^{2}}\left(\varepsilon_{; i} \varepsilon_{; k}^{*}+\varepsilon_{; i}^{*} \varepsilon_{; k}-g_{i \hbar} \varepsilon_{; r} \varepsilon^{* ; r}\right) .
\end{aligned}
$$

These equations can be derived from the Lagrangian

$$
L=R+\frac{1}{2} \frac{\varepsilon_{; i} \varepsilon^{* ; i}}{(\operatorname{Re} \varepsilon)^{2}}
$$

by using the variational principle.

Sometimes it is more convenient to introduce the function $\xi$ (Ref. [3], see also Section 4) by

$$
\xi=\frac{1+\varepsilon}{1-\varepsilon} .
$$

We remark that the stress tensor is expressed in terms of the field variable $\xi$ as follows:

$$
T_{i k}=-\left(\xi \xi^{*}-1\right)^{-2}\left(\xi_{; i} \xi_{; k}^{*}+\xi_{; i}^{*} \xi_{; k}-g_{i k} \xi_{; r} \xi^{* ; r}\right)
$$

\section{Invariant Classification}

As is well known, the Weyl tensor $C_{i j k l}$ vanishes in $V_{3}$, so that the relationship between the curvature tensor and Ricci tensor reads [5]: $R_{i j k l}=-g_{i l} R_{j k}+g_{i k} R_{j l}-g_{j l} R_{i l}+g_{j l} R_{i k}+\frac{1}{2} R\left(g_{i l} g_{j k}-g_{i k} g_{j l}\right)$. 
The classification with respect to the algebraic structure of the curvature tensor is therefore completely equivalent in $V_{3}$ to that of $R_{i j}[6]$. We shall deal with the tracefree part $P_{i}^{k}$ of the Ricci tensor:

$$
P_{i}^{k}=R_{i}^{k}-\frac{1}{3} \delta_{i}^{k} R .
$$

It is convenient to use in the calculations the line element (2). In this coordinate system we find:

$$
\left[P_{i}^{k}\right]=\frac{e^{-2 \gamma}}{3}\left[\begin{array}{ccc}
\gamma_{11}+\gamma_{22}-3 \gamma_{1} / \varrho & -3 \gamma_{2} / \varrho & 0 \\
-3 \gamma_{2} / \varrho & \gamma_{11}+\gamma_{22}+3 \gamma_{1} / \varrho & 0 \\
0 & 0 & -2 \gamma_{11}-2 \gamma_{22}
\end{array}\right]
$$

The eigenvalue-problem

$$
P_{i}^{k} v^{i}=\lambda v^{k}
$$

leads to the following characteristic equation:

$$
\left[2\left(\gamma_{11}+\gamma_{22}\right)+\Lambda\right]\left[\left(\gamma_{11}+\gamma_{22}-\Lambda\right)^{2}-9\left(\gamma_{1}^{2}+\gamma_{2}^{2}\right) / \varrho^{2}\right]=0
$$

where $\Lambda=3 e^{2 \gamma} \lambda$. The solutions of Eq. (21) are:

$$
\begin{aligned}
& \Lambda_{0}=-2\left(\gamma_{11}+\gamma_{22}\right), \\
& \Lambda_{ \pm}=\gamma_{11}+\gamma_{22} \pm 3 \varrho^{-1}\left(\gamma_{1}^{2}+\gamma_{2}^{2}\right)^{1 / 2} .
\end{aligned}
$$

Using the field equations (12), (13), the eigenvalues can be written in an invariant form:

$$
\left.\begin{array}{l}
\lambda_{0}=\frac{1}{6}\left|\frac{\nabla \varepsilon}{\operatorname{Re} \varepsilon}\right|^{2} \\
\lambda_{ \pm}=-\frac{1}{12} \frac{|\nabla \varepsilon|^{2} \mp 3|\nabla \varepsilon \nabla \varepsilon|}{(\operatorname{Re} \varepsilon)^{2}}
\end{array}\right\}
$$

We have $\lambda_{0}+\lambda_{+}+\lambda_{-}=0$ because $\left[P_{i}^{k}\right]$ is tracefree.

If we restrict ourselves to physically realistic spaces, for which the asymptotic conditions (4) hold, we have asymptotically:

$$
\lambda_{0}=\lambda_{+}=-2 \lambda_{-}=\frac{2}{3} \frac{m^{2}}{r^{4}} .
$$

The type of the Ricci tensor is asymptotically degenerate $(D)$. The alternative possibility is that $\lambda_{0} \neq \lambda_{+}$, when the type is called general $(G)$. All other possibilities, as e.g. $\lambda=0$ are excluded by the asymptotic conditions (4) for physically interesting spaces.

Now we have shown that the axially symmetric stationary gravitational fields may be classified in an invariant manner, with respect to the algebraic structure of their Ricci tensor in the corresponding space $V_{3}$. The possible types are $D$ and $G^{1}$.

1 The present classification differs from that of Levy [1]. 
The necessary and sufficient condition of the degeneracy is learned from Eq. (23) :

$$
f_{1} \varphi_{2}-f_{2} \varphi_{1}=0 .
$$

So the class of the type $D$ vacuum solutions consists only of the static Weys spaces [5] and of Papapetrou's solutions [2]; for the latter we have

$$
\Lambda_{0}=\Lambda_{+}=\frac{1}{2}\left(\chi_{12}^{2}+\chi_{22}^{2}\right)
$$

where $\chi$ is an arbitrary harmonic function. However, we recall the well known fact that Papapetrou's solutions, not satisfying the asymptotic conditions (4), are physically unacceptable (the mass monopole term is lacking).

All other relevant spaces, among others the Kerr metric [7], being now the only known solution of the field Eqs. (12), (13) which can really represent the gravitational field of a bounded rotating source, are of type $G$. For the Kerr solution one has

$$
\left.\begin{array}{l}
\lambda_{0}=\frac{2}{3} \frac{m^{2}}{\left(r^{2}-2 m r+a^{2} \cos ^{2} \theta\right)^{2}}\left(1+\frac{2 a^{2} \sin ^{2} \theta}{r^{2}-2 m r+a^{2} \cos ^{2} \theta}\right), \\
\lambda_{ \pm}=-\frac{1}{3} \frac{m^{2}}{\left(r^{2}-2 m r+a^{2} \cos ^{2} \theta\right)^{2}}\left(1 \mp 3+\frac{2 a^{2} \sin ^{2} \theta}{r^{2}-2 m r+a^{2} \cos ^{2} \theta}\right)
\end{array}\right\}
$$

where the functions $r(\varrho, z)$ and $\theta(\varrho, z)$ are defined by the relations

$$
\begin{aligned}
& \varrho=\left(r^{2}-2 m r+a^{2}\right)^{1 / 2} \sin \theta, \\
& z=(r-m) \cos \theta .
\end{aligned}
$$

It is seen from (27), (28) also that the space becomes asymptotically type $D$.

\section{The Electrovac Problem}

As we shall see, the extension of 3-dimensional "relativity" to electrovac spaces yields rather complicated mathematical expressions, although the results are very similar to those in the absence of electromagnetism. The only important difference is that the type $D$ electrovac metrics are not all known.

Our notation is in agreement with Ref. [8]: $A_{\mu}$ stands for the electromagnetic 4-potential and the field variables $A_{3}^{\prime}, \Phi, \varphi, \varepsilon$ used here are defined by the relations

$$
\begin{aligned}
\hat{n} \times \nabla A_{3}^{\prime} & =\varrho^{-1} f\left(\nabla A_{3}-\omega V A_{4}\right), \\
\Phi & =A_{4}+i A_{3}^{\prime}, \\
\hat{\imath} \times \nabla \varphi & =\varrho^{-1} f^{2} \nabla \omega-2 \hat{n} \times \operatorname{Im}\left(\Phi^{*} \nabla \Phi\right), \\
\varepsilon & =f-|\Phi|^{2}+i \varphi .
\end{aligned}
$$


The unit vector $\hat{n}$ is seen to be the Killing vector of the space $V_{3}$ with the line element (2). In properly chosen units the first group of the field equations which govern the axially symmetric stationary electrovac spaces is as follows:

$$
\left.\begin{array}{l}
\left(\operatorname{Re} \varepsilon+|\Phi|^{2}\right) \Delta \varepsilon=\left(V_{\varepsilon}+2 \Phi^{*} \nabla \Phi\right) \nabla \varepsilon \\
\left(\operatorname{Re} \varepsilon+|\Phi|^{2}\right) \Delta \Phi=\left(\nabla \varepsilon+2 \Phi^{*} \nabla \Phi\right) \nabla \Phi
\end{array}\right\}
$$

To any solution of the field Eqs. (12), (13) it is possible to find its "electrovac pair" for which $\varepsilon=\frac{\xi^{\prime}-1}{\xi^{\prime}+1}$ is an analytic function of $\Phi$. Then one has [8]

$$
\varepsilon=1-2 \Phi / q,
$$

where $q$ is a complex constant. The field Eqs. of Sec. 2 are formally retained by denoting $\xi=\left(1-q q^{*}\right)^{-1 / 2} \xi^{\prime}$.

It will prove advantageous to introduce the complex 3 -vectors

and

$$
G=\frac{1}{2} \frac{V \varepsilon+2 \Phi^{*} \nabla \Phi}{\operatorname{Re} \varepsilon+|\Phi|^{2}}
$$

$$
\boldsymbol{H}=\left(\operatorname{Re} \varepsilon+|\Phi|^{2}\right)^{-1 / 2} \nabla \Phi .
$$

The second group of electrovac field equations is now equivalent to the "gravitational equations" in $V_{3}$ having the line element (2) and the stress tensor of the form

$T_{i k}=-\left\{\left(G_{i} G_{k}^{*}+G_{i}^{*} G_{k}\right)-\left(H_{i} H_{k}^{*}+H_{i}^{*} H_{k}\right)-g_{i k}\left(G^{r} G_{r}^{*}-H^{r} H_{r}^{*}\right)\right\}$.

One easily finds now that the corresponding Lagrangian is

$$
L=R+2\left(G^{r} G_{r}^{*}-H^{r} H_{r}^{*}\right) .
$$

The field Eqs. (33) together with the integrability conditions for $\varepsilon$ and $\Phi$ yield a complex non-linear "electrodynamics" for $\boldsymbol{G}$ and $\boldsymbol{H}$ :

$$
\begin{aligned}
(\nabla-\boldsymbol{G}) \boldsymbol{G} & =\boldsymbol{H}^{*} \boldsymbol{H}-\boldsymbol{G}^{*} \boldsymbol{G} \\
\nabla \times \boldsymbol{G} & =\boldsymbol{H}^{*} \times \boldsymbol{H}-\boldsymbol{G}^{*} \times \boldsymbol{G} \\
(\nabla-G) \boldsymbol{H} & =\frac{1}{2}\left(\boldsymbol{G}-\boldsymbol{G}^{*}\right) \boldsymbol{H} \\
\nabla \times \boldsymbol{H} & =-\frac{1}{2}\left(\boldsymbol{G}+\boldsymbol{G}^{*}\right) \times \boldsymbol{H} .
\end{aligned}
$$

The covariant vectorial product $\boldsymbol{A} \times \boldsymbol{B}$ is defined as follows

$$
(\boldsymbol{A} \times \boldsymbol{B})_{i} \stackrel{\text { def }}{=} \varepsilon_{i j k} A^{j} B^{k} \sqrt{g} .
$$

An apparent property of the field equations is their invariance against the constant phase-shift $\boldsymbol{H} \rightarrow e^{i c} \boldsymbol{H}$. This phase-shift corresponds to a duality rotation of the electromagnetic field. Consequently, in the 
static limit both $\boldsymbol{G}$ and $\boldsymbol{H}$ may be taken real. Another important special case results if $\boldsymbol{H}$ vanishes (absence of electromagnetism).

We put down here the eigenvalues of the Ricci tensor also:

$$
\begin{aligned}
& \lambda_{0}=\frac{2}{3}\left(|\boldsymbol{G}|^{2}-|\boldsymbol{H}|^{2}\right), \\
& \lambda_{ \pm}=-\frac{1}{3}\left(|\boldsymbol{G}|^{2}-|\boldsymbol{H}|^{2}\right) \pm\left\{\left|G^{2}\right|^{2}+\left|H^{2}\right|^{2}-2|\boldsymbol{G} \boldsymbol{H}|^{2}+2\left|G^{2} H^{2}-(\boldsymbol{G} \boldsymbol{H})^{2}\right|\right\}^{1 / 2}
\end{aligned}
$$

Hence the condition of degeneracy $\lambda_{0}=\lambda_{+}$is seen to be

$$
\left(\boldsymbol{G}^{*} \times \boldsymbol{G}-\boldsymbol{H}^{*} \times \boldsymbol{H}\right)^{2}=-4|\boldsymbol{G} \times \boldsymbol{H}|^{2} .
$$

If (42) holds, the space is type $D$, otherwise $G$. For static spaces (42) simplifies to requiring that $\boldsymbol{G}$ and $\boldsymbol{H}$ are parallel vectors. Known examples of static electrovac spaces are the type $D$ solutions of WEYL [9], for which $\varepsilon=\varepsilon(\Phi)$ is assumed, or the space with the line element

$$
\begin{aligned}
d s^{2}= & -N^{2}\left(r^{2}-a^{2} \cos ^{2} \theta\right)^{2}\left[(r-m)^{2}-\left(a^{2}+m^{2}\right) \cos ^{2} \theta\right]^{-3} \\
& \times\left[d r^{2}\left((r-m)^{2}-\left(a^{2}+m^{2}\right)\right)^{-1}+d \theta^{2}\right]-N^{-2}\left[(r-m)^{2}-\left(a^{2}+m^{2}\right)\right] \\
& \times\left[r^{2}-a^{2} \cos ^{2} \theta\right]^{2} \cdot \sin ^{2} \theta d \Phi^{2}+N^{2}\left(r^{2}-a^{2} \cos ^{2} \theta\right)^{-2} d t^{2}
\end{aligned}
$$

and magnetic potential

$$
A_{3}=2 \operatorname{mar} \sin ^{2} \theta / N
$$

(a, $m$ are constants, $\left.N=(r-m)^{2}-m^{2}-a^{2} \cos ^{2} \theta\right)$.

This latter solution was obtained from the Kerr metric by using an accidental symmetry of the axially symmetric spaces [10] and is of type $G$.

The metric found by Newman et al. $[8,11]$ is the "electrovac pair" of the Kerr solution and is also type $G$. One can construct the "electrovac pairs" of Papapetrou's stationary solution also, which are then of type $D$. These metrics, together with some other new solutions of the electrovac field Eqs. (39) will be dealt with in more details in a subsequent paper.

\section{Summary}

After the 3-covariant formulation of the stationary axisymmetric gravitational field problem, many of the succesful methods of general relativity, as those based on the algebraic features of the curvature tensor (Section 3) or maybe on optical properties, can be applied to this particular problem. These may prove useful in finding new axially symmetric stationary spaces.

On the other hand, our procedure may shed some light on the question, whether general relativity can fully be "geometrized" because it gives an example when the material field becomes part of the metric in a higher dimensional empty space theory.

Further investigations are needed in order to find the possible extensions of our results to more general gravitational fields. 
Acknowledgements. The author would like to thank Dr. F. KÁRoLYHÁzy for valuable discussions and the entire theoretical group of Central Research Institute for Physics for helpful comments.

\title{
Literature
}

1. Levy, H.: Nuovo Cimento, „56B, 253 (1968).

2. Papapetrou, A.: Ann. Physik 12, 309 (1933).

3. Ernst, F. J.: Phys. Rev. 167, 1175 (1968).

4. Papapetrou, A.: Ann. Inst. Poincaré 4, 83 (1966).

5. Petrov, A. Z.: New methods in the theory of general relativity (russian). Moscow: Nauka 1966.

6. For the classification of the Ricci tensor in four dimensions, see. Ref. [5], p. 378.

7. KerR, R.: Phys. Rev. Letters 11, 237 (1963).

8. Ernst, F. J.: Phys. Rev. 168, 1415 (1968).

9. Weyl, H.: Ann. Physik. 54, 117 (1917).

10. Bonnor, W.: Z. Physik. 190, 444 (1966); see also PerJés, Z.: Nuovo Cimento $55 \mathrm{~B}, 600$ (1968).

11. Newman, E.T., E. Couch, K. Chinnapared, A. Exton, A. Prakash, and R. Torrence: J. Math. Phys. 6, 918 (1956).

\author{
Z. PeRJÉS \\ Hungarian Academy of Sciences \\ Central Research Institute for Physics \\ Budapest 114, Hungary
}

